\title{
Sanktionspolitik gegen Russland
}

Die Geschehnisse in der Ostukraine haben die internationalen wirtschaftlichen und politischen Konfrontationen zwischen Russland und den westlichen Industrieländern seit 2014 massiv verschärft. Um Druck auf Russland auszuüben und eine Verhaltensänderung herbeizuführen, verhängten auf Bestreben der USA und EU mehrere westliche Länder seit dem Frühjahr 2014 stufenweise wirtschaftliche und politische Sanktionen gegenüber Russland. Russland konterte mit Gegenmaßnahmen und Importbeschränkungen auf Agrarerzeugnisse und Nahrungsmittel von diesen Ländern. Nun drohen die westlichen Länder bei einer weiteren militärischen Aggression Russlands gegen die Ukraine, die Sanktionen zu verschärfen. Neben einer Erweiterung des bestehenden Instrumentariums stehen Finanzsanktionen sowie Restriktionen gegen die staatsnahe Industrie, vor allem die Öl- und Gasindustrie sowie Bergbauunternehmen, im Raum. Beide Optionen wären mit massiven wirtschaftlichen Folgen für alle Beteiligten verbunden. Folgende Fragen drängen sich auf:

Wie wirksam sind Sanktionen? Es ist gut belegt, dass umfassende und von breiten Koalitionen getragene Handelssanktionen starke Effekte auf den Handel zwischen den Konfliktparteien haben (Felbermayr et al., 2021). Diese handelszerstörenden Effekte sind gut quantifizierbar, da die empirische Gravitationsgleichung es erlaubt, glaubwürdige Kontrollgruppen zu identifizieren. Ob Sanktionen auch die politischen Ziele eines Politikwechsels erreichen, ist empirisch nicht abschließend gezeigt. Einerseits sind Drohungen mit Sanktionen nicht gut beobachtbar (Morgan et al., 2009), andererseits fehlt das Kontrafaktum. Klar ist, dass die starke Zunahme von Wirtschaftssanktionen seit 1950 per se einen Beleg für den Misserfolg dieser Politik darstellt, denn theoretisch sollte die Drohung ausreichen, die angestrebten Ziele zu erreichen. Müssen tatsächlich Sanktionen verhängt werden, dann war die Drohung offenbar nicht glaubwürdig oder stark genug. Wenn es so weit kommt, zeigt die Global Sanctions Data Base (Felbermayr et al., 2020), dass nur rund ein Drittel der von 1950 bis 2019 erfassten Sanktionsperioden als erfolgreich dokumentiert wird. Daher ist es paradox, dass sich dieses politische Instrumentarium zunehmender Beliebtheit erfreut. Den von der EU angestrebten Regimewandel in Russland zu bewirken, erscheint bei der wirtschaftlichen Größe und Beziehungen zu anderen Ländern sowie angesichts hoher Devisenreserven und Rohstoffvorkommen äußerst schwer. Darüber hinaus ist auch die ungleiche wirtschaftliche Betroffenheit durch die Sanktionen innerhalb der EU-Länder ein politisches Dilemma. Die untergeordnete Bedeutung der USA im Handel mit Russland und die damit kaum vorliegenden ökonomischen Nachteile aus den bisherigen Sanktionen stellen ein politisches Problem dar. Im Fall Russlands müssten auch China und Indien als wichtige Handelspartner mitwirken, um wirtschaftlichen Druck auszuüben. Zugleich ist das Maß an smarten Sanktionen gegen Russland weitgehend ausgeschöpft und damit das Drohpotenzial weit kleiner.

Welche Werkzeuge liegen vor? Um im Fall einer Militäroperation in der Ukraine eine rote Linie aufzuzeigen, bedarf es kraftvoller, gezielter Sanktionen, die auch in Russland ihre Wirkung entfalten. Derzeit werden von der EU und den USA vor allem der Ausschluss Russlands vom internationalen Zahlungsverkehr SWIFT sowie ein weitgehender Verzicht der EU auf russische Gas- und Öllieferungen diskutiert. Ebenso hat die USA angekündigt, Russland an der Verwendung des US-Dollars zu hindern und den Technologieexport zu beschränken, wodurch Russland weitgehend von der Versorgung mit Elektronikprodukten abgeschnitten wäre. Auch eine weitere Einschränkung des Zugangs zu russischen Anleihen steht als Druckmittel im Raum. Alle Optionen sind für die Beteiligten sehr kostspielig, vor allem für Russland und die EU, die intensivere Handels- und Wirtschaftsbeziehungen pflegen. Mit dem Ausschluss aus SWIFT wäre für die von Rohstoffexporten abhängige russische Wirt-

(C) Der/die Autor:in 2022. Open Access: Dieser Artikel wird unter der Creative Commons Namensnennung 4.0 International Lizenz veröffentlicht (creativecommons.org/licenses/by/4.0/deed.de).

Open Access wird durch die ZBW - Leibniz-Informationszentrum Wirtschaft gefördert.

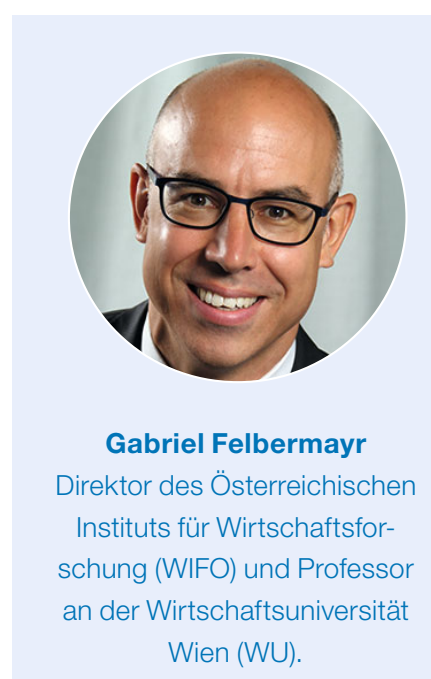


schaft der Waren- und Zahlungsverkehr mit dem Ausland deutlich erschwert. Auch für die EU wäre es fast unmöglich, ohne Umwege über andere Länder Handel mit Russland zu treiben bzw. Gasimporte abzuwickeln. Ein Stopp der Gaslieferungen in die EU würde vermutlich Produktionsunterbrechungen in Industrieunternehmen sowie weitaus höhere Energiepreise zur Folge haben. Die Kosten wären schmerzhaft, aber wirtschaftlich verkraftbar.

Wie hoch ist die gegenseitige Abhängigkeit? Gerade im Hinblick auf die Energieversorgung ist die EU verwundbar. 2020 stammten rund $44 \%$ der EU-Gasimporte aus Russland, und alternative Gaslieferanten, wie z.B. Flüssiggaslieferungen aus den USA, könnten einen kompletten Einfuhrstopp nicht kompensieren. Gleichzeitig basiert die russische Wirtschaft weitgehend auf dem Export von Öl und Gas und ist stark von ausländischem Kapital abhängig. Etwa ein Drittel des russischen Staatshauhalts beruht auf den Gasexporten. Eine Unterbrechung der Gaslieferungen in die EU hätte massive Auswirkungen auf die russische Wirtschaft. Im Banken- und Finanzsektor sind die relativen Abhängigkeiten asymmetrischer. Während die direkte Abhängigkeit der führenden Industrienationen von Russland hier keine Systemrelevanz aufweist, ist Russland relativ stark auf die globalen Finanzmärkte und reibungslos funktionierende Bank- und Zahlungsverbindungen angewiesen. Die Pläne Chinas und Russlands, ein alternatives Zahlungssystem aufzubauen, sind zwar bereits teilweise umgesetzt, erlauben aber im Fall des russischen Systems (System for Transfer of Financial Messages, SPFS) derzeit nur Transaktionen innerhalb Russlands.

Wie viel kosten Sanktionen? Kaum eine politische Maßnahme ist nicht mit Kosten verbunden. Doch auch die Alternative - geopolitische Spannungen - ist mit Kosten verbunden und eine potenzielle Militärintervention Russlands in die Ukraine würde den Handel der EU mit Russland nahezu zum Erliegen bringen. Wenngleich der russische Markt groß ist, gingen 2020 nur rund 1,65 \% der EU-Exporte nach Russland. Ebenso stammten auch nur 2,1 \% der EU-Importe aus Russland. Gleichzeitig ist die EU für Russland der wichtigste Markt. Infolge der 2014 verhängten Sanktionen sowie des generellen Wirtschaftseinbruchs in Russland, bedingt durch einen massiven Rohölpreisverfall und eine starke Abwertung des Rubels, verzeichneten alle EU-Länder und auch Russland massive Exporteinbußen. Für die EU können rund $40 \%$ des beobachteten Exportrückgangs auf die Sanktionen und Gegensanktionen zurückgeführt werden (Christen et al., 2016). Die volkswirtschaftlichen Kosten betragen in Russland etwa 35 Mrd. Euro (ca. $2 \%$ des BIP) und in der EU knapp 20 Mrd. Euro (ca. 0,2 \% des BIP; Fritz et al. 2017), wenngleich die regionale Betroffenheit innerhalb der EU-Länder sehr heterogen ist (Chowdhry et al., 2020). Im Gegensatz dazu sind die ökonomischen Folgen für die USA deutlich milder. Diese ungleiche Betroffenheit ist ein politisches Problem, vor allem in der Frage um breite Koalitionen, gemeinsame Ziele und eine wirksame Drohung.

Zusammenfassend haben Sanktionsdrohungen gegenüber Russland wohl nur eine Chance auf Erfolg, wenn die EU glaubwürdig signalisiert, dass sie im Ernstfall bereit wäre, mit hohen wirtschaftlichen Kosten die Gaslieferungen zu unterbrechen. Dazu ist die EU, die in Fragen der Außenpolitik Einstimmigkeit erfordert, vermutlich nicht in der Lage.

Elisabeth Christen,

Gabriel Felbermayr

Österreichisches Institut für

Wirtschaftsforschung (WIFO), Wien

\section{Literatur}

Christen, E., O. Fritz, G., Streicher und J. Hinz (2016), Auswirkungen der Wirtschaftssanktionen der EU und Russlands auf Wertschöpfung und Beschäftigung in Österreich und der EU, WIFO.

Chowdhry, S., G. Felbermayr, J. Hinz, K. Kamin, A.-K. Jacobs und H. Mahlkow (2020), The Economic Costs of War by Other Means, Kiel Policy Brief, 147.

Felbermayr, G., A. Kirilakha, C. Syropoulos, E. Yalcin und Y. Yotov (2020), The Global Sanctions Data Base, European Economic Review, 129(C).

Felbermayr, G., C. Morgan, C. Syropoulos und Y. Yotov (2021), Understanding Economic Sanctions: Interdisciplinary Perspectives on Theory and Evidence, European Economic Review, 135(C).

Fritz, O., E. Christen, J. Hinz und F. Sinabell (2017), Russia's and the EU's Sanctions Economic and Trade Effects, Compliance and the Way Forward, Research Report for the European Parliament.

Morgan, T., N. Bapat und V. Krustev (2009), The Threat and Imposition of Economic Sanctions, 1971-2000, Conflict Management and Peace Science, 26(1), 92-110. 